\title{
Remote health monitoring of elderly through wearable sensors
}

\author{
Mohammed Al-khafajiy ${ }^{1} \cdot$ Thar Baker $^{1}$ (D) . Carl Chalmers ${ }^{1}$. \\ Muhammad Asim ${ }^{2} \cdot$ Hoshang Kolivand ${ }^{1} \cdot$ Muhammad Fahim ${ }^{3} \cdot$ Atif Waraich $^{1}$
}

Received: 30 September 2018 / Revised: 1 December 2018 / Accepted: 26 December 2018 /

Published online: 24 January 2019

(C) The Author(s) 2019

\begin{abstract}
Due to a rapidly increasing aging population and its associated challenges in health and social care, Ambient Assistive Living has become the focal point for both researchers and industry alike. The need to manage or even reduce healthcare costs while improving the quality of service is high government agendas. Although, technology has a major role to play in achieving these aspirations, any solution must be designed, implemented and validated using appropriate domain knowledge. In order to overcome these challenges, the remote real-time monitoring of a person's health can be used to identify relapses in conditions, therefore, enabling early intervention. Thus, the development of a smart healthcare monitoring system, which is capable of observing elderly people remotely, is the focus of the research presented in this paper. The technology outlined in this paper focuses on the ability to track a person's physiological data to detect specific disorders which can aid in Early Intervention Practices. This is achieved by accurately processing and analysing the acquired sensory data while transmitting the detection of a disorder to an appropriate career. The finding reveals that the proposed system can improve clinical decision supports while facilitating Early Intervention Practices. Our extensive simulation results indicate a superior performance of the proposed system: low latency (96\% of the packets are received with less than 1 millisecond) and low packets-lost (only $2.2 \%$ of total packets are dropped). Thus, the system runs efficiently and is cost-effective in terms of data acquisition and manipulation.
\end{abstract}

Keywords Internet of things - Ambient assisted living $\cdot$ Healthcare $\cdot$ Sensor network · Real-time monitoring · Wireless sensor networks

\section{Introduction}

Technology has become an essential and integral component in modern day living and influences the way in which we all live and work. For the most part technology has a positive impact as its helps us to manage and control our daily routines. However, modern day

Mohammed Al-khafajiy

M.D.Alkhafajiy@2016.ljmu.ac.uk

Extended author information available on the last page of the article. 
technology has an important role to play in other domains such as overcoming the many challenges facing health and social care. Ambient Assistive Living (AAL) is the concept of generating new living spaces that combine social environments with the latest technology to produce products and services that significantly enhance the quality of life of the occupant. Many areas of research and technology contribute to assistive solutions, with numerous trials undertaken to assess their feasibility. The application of several domains has been exploited to enable and improve the use of assistive technologies within the household. The collection and integration of such technologies is often referred to as Ambient Intelligence or AAL, which aims to support people by enabling them to achieve their everyday objectives. AML is not only regarded as the integration of various technologies (i.e. sensors, computing etc.) and related domains (i.e. computer science, engineering, medicine and social sciences) but focuses on incorporation and applicability of such technologies. The main function of any system is the ability to identify human activities from the acquired sensory data. This is achieved by applying meaning (which is usually in the form of medical insight) to observations.

The underpinning concept of many AAL technologies is the Internet of Things (IoT) paradigm. IoT is a framework that enables developers to connect several devices, systems and technologies together to achieve certain tasks [14] such as health monitoring. The rapid growth of wearable sensors (e.g., fitness trackers, wearable biometric sensors etc.) changes the way we collect and analyse data [4, 6]. A large proportion of IoT technology directly builds on the motivation of monitoring our daily activities (e.g., monitoring steps and diets throughout the day). For instance, using a smart wristband, which is commonly used for monitoring health and fitness. Smart phones which are based on the electrocardiogram can be used to assess and analyses heart's condition [32]. The Brain Sensing Headband monitors brain activities and transmits information to a computer, smart-phone, or tablet via Bluetooth [23]. Wearable sensor technologies and IoT have great potential to improve our lifestyle, such as providing healthcare monitoring systems which are responsible for tracking and managing our health and fitness. Additional features such as real-time communication enables the data to be transmitted and analysed by healthcare providers while providing the means to identify and act upon worrying behaviour or symptoms. Raising the efficiency and overall cost effectiveness of healthcare is one of the vital goals of modern society.

The worldwide population has increased significantly which is largely due to the advancement in medicine while the number of elderly people who need more care $[35,36]$ is increasing. For many countries, the emergence of an ageing population is fast becoming an increasing public health concern. Healthcare costs are continuously rising and the quality of services does not meet the needs of modern society. Remote real-time health monitoring provides one possible solution to overcoming these challenges. Constantly monitoring the health of elderly people via wearable devices and fitness trackers proved different avenues in facilitating Early Intervention Practices EIP and reducing release rates. Surveys undertaken in Europe and the United States [36] indicate that patients prefer to undergo treatment and healthcare monitoring in their own homes rather than being hospitalised. Monitoring patients remotely have several advantages, which include EIP, reduce relapse rates, while reduction in costs patient and convenience become a possible outcome [30].

Governments and healthcare organisations spend millions of pounds every year in providing high quality of services for healthcare sectors [36]. Monitoring patient's vital signs such as temperature, blood pressure and heart rate are one of the major aspects of today's 
healthcare services [17]. Bed blocking [16, 27, 40], where a patient is medically ready to be discharged but is delayed because of inadequate care, support and rehabilitation services outside hospitals has become increasingly common within the healthcare sector. Until recently, patients were monitored manually in clinics and or hospitals for long periods while keeping accurate reports and records for future use by doctors and other clinicians if needed [15]. However, by combining multiple technologies such as smart-phone applications, wearable devices and sensors, a patient's well-being can be accurately monitored remotely.

Our research motivations come from the interest in enhancing the living facilities in general, and our healthcare in particular. Healthcare services in our modern societies become capable to meet the need of individuals, because, on one hand, the significant growth of population leads to more consumers in our current and static healthcare systems. On the other hand, healthcare investment in modern technologies to handle these populations is still limited. Moreover, similarly to other people we are substantially care about our health and interested in constantly measuring our physical activities any-where at any-time using various healthcare and fitness trackers devises such the smart watches. In addition, while we are doing our day by day jobs we care about our elder and disabled people health when we leave them home alone for a long period. Therefore, it is becoming essential to engage technologies such as healthcare sensors and wearable with our healthcare systems, in order to have a safer and convenient environment for everyone to live in.

The growing elderly population, accompanied by the increasing prevalence of chronic diseases associated with ageing, will have profound implications for the health care system for decades to come [13]. Therefore, we are proposing a system which enables continuous monitoring for elderly people health in real-time to prevent chronic diseases, thus preventing hospitalisation that burden the healthcare systems and costs. This paper presents a framework which utilises a smart-phone app and Wearable Sensors for Smart Healthcare Monitoring System (SW-SHMS) for elderly people. The system accumulates patient's physiological data via wearable sensors (i.e., pulse, oxygen etc.) of elderly people in real-time. The data is transmitted to a data repository, where it will be stored and checked for any abnormality. Thus, any detection of disorder in a patient's vitals will be reported to patient's doctors and/or hospital in real-time to act on quickly and prevent a number of problems, such as, a sudden heart attack. Technologies are capable of providing patients physiological data from their locations to physicians anywhere in real-time, therefore, enabling remote remediation [18]. For example, data such as blood oxygen saturation, heart-rate, and blood pressure can be measured via wearable devices and transmitted from patients locations to their doctors in real-time. This enables doctors and patients to communicate remotely. Providing such a system that can effectively monitor an elderly person physiological activity at regular intervals could detect diseases and other complications earlier [35]. Especially, in the case of elderly people who are much likely to have a disorder in their physiological data [36]. It is an utmost necessity to develop new methods and technologies in order to improve health services for the elderly community at an affordable price with ease of use while ensuring maximum comfort and independence.

The rest of the paper is organised as follows: related work for the proposed system is discussed in Section 2. The SW-SHMS system and its associated components are described in Section 3. A prototype system for the SW-SHMS is described in Section 4. The results from the implantation are presented in Section 5. Finally, the paper is concluded in Section 6 with a potentials for future directions of this work. 


\section{Related work}

Recent advances in research and technology have broadened the horizons of the AAL platforms committed to assist ageing adults in their own residence. Wearable sensors have played a crucial role in the development of non-invasive strategies to monitor people activities. The purpose of this section is a two-fold: (i) reviews of similar systems/applications; (ii) The state of the art technologies in the field of sensors in remote monitoring.

\subsection{State-of-the-art}

The increase in lifestyle related disease along with the increase of the ageing population it becomes crucial to develop systems that facilitate the monitoring of health status independent at home and work. One important trend is that both the acquisition and processing of physiological data are moving out from the hospitals, allowing patients to stay at home or at work [33].

Several research groups and industry have initiated projects in the AAL area, for instance My-Heart [25], an EU FP6 project with Philips Research group focusing on vital sign monitoring to save lives by providing earlier diagnoses, closer monitoring and a means to help people manage any heart problems. In addition, HeartCycle project $[11,24]$, also coordinated by Philips, provide a closed-loop disease management solution being able to serve both Heart Failure (HF) patients and Coronary Heart Disease (CHD) patients, including possible co-morbidities hypertension, diabetes and arrhythmias. This has been achieved by multi-parametric monitoring and analysis of vital signs and other measurements. In the industry, the Continua Health Alliance [33, 44], which started in 2006 to enable an interoperable personal telehealth ecosystem, has become a major force in the personal telehealth domain, and over a dozen interoperable products have already been certified. Continua [44] has also made great progress in defining interoperability for the LANand WANinterfaces and now enables end-to-end interoperability [33].

Furthermore, Several projects have been conducted on the AAL framework and we have arranged them under their main functions. We first enumerate a group of projects focused on allowing people to age at home, being some of them funded by the Assisted Living Joint Programme ${ }^{1}$ projects fund [3]. The project Aware Home $[3,12]$ uses a wide variety of sensors, covering from specifically designed smart floors to more typical video and ultrasonic sensors, together with social robots to monitor and help older adults [3, 10]. The Ubiquitous Home Project [45] is also centred in residents monitoring through a robot, which includes a dialogue based interface and operates as an intermediate platform between the smart home and the end users [3]. In addition, some projects have focused on the monitoring patients suffering from a chronic disease, such as CASAS project [39], which use the smart-homes environment to monitor patients suffering from dementia, which is similar to ENABLE project [1] with the goal of giving them more autonomy in their lives. While, DOMUS [7] and IMMED [28] projects focus on behaviour recognition for patients suffering from Alzheimer disease [3].

Grenoble Health Smart Home [21] proposed a set of tools to measure patients activity in hospitals via several indicators of their activities in daily living, mobility, and repartition of stays and displacements. In the Gloucester Smart House project [5], a telehealth system was

\footnotetext{
${ }^{1}$ EU Commission. Active and Assisted Living Programme. ICT for Ageing Well in a digital world. Available at: www.aal-europe.eu.
} 
designed, based on lifestyle monitoring, with the pretension of continuously gathering information about the patients activity during daily routines. Some of these systems also sample biomedical constants, like in theWelfare Techno House project [43], which collects automated electrocardiogram measurements. In the Aging in Place project [38], residents are tested for urinary tract infections and alerts are sent to the caregiver using a sensor network. The goal was to detect signs of illness earlier than traditional health care assessment.

\subsection{Related technologies}

The development of new and innovate technologies in modern day life and their ability to enhance our daily activities has lead researches to focus on their use within clinical domains. The improvement of healthcare systems is gaining significant focus from both researchers and industry alike, by providing more convenient and automated healthcare products [19]. Research on remote monitoring technology is increasing due to rising in healthcare costs $[35,36]$ and the significant increase of ageing population [15]. Therefore, developing new innovative technologies to solve healthcare issues became one of growing fields. In this section, we will investigate several recent researches and related work to the this research.

One of the smart home experiments to produce a smart environment for healthcare in [36], presented a cloud-based smart home called CoSHIE. This system collects physiological data through wearable sensors. Minh et al. [36], use a non-invasive sensor to gather information about patient's daily activities. The case study in this work proves that this approach is effective to collect contextual information to study patient's behavioural changes and recovery processes at their homes. The sensor collects data and sends it to cloud via home getaway. CoSHIE cloud has a number of layers which include the hybrid storage that includes the relational (MySQL), and non-relational (NoSQL) data-centre. The authors intended to sort data such as name, sex, diagnosis, fluid intake and Spo2. All sensors data are stored in the non-relational databases, while other information is saved in the relational databases. One of the limitations of this system is the lack of direct monitoring for patients from their doctors or caregiver in case of disorder or emergency. There are several works on using micro-controller such as Arduino and Raspberry Pi for remote monitoring in healthcare, for instance improving healthcare using Raspberry Pi to help in remote monitoring by Gupta et al. [17], focused on monitoring one parameter which is ECG sign. Raspberry Pi was use as getaway to collect the ECG data from different wearable machines and send it to the databases. Thus, in case any change in the data happens, the Raspberry Pi will update the databases using MySQLdb. In addition, a wireless network (GSM) used to send an alert to doctor in case of emergency. The data will be available to access by authorised people through web application.

Wireless sensor network [2,9] is presented to the collection of physical data such as blood pressure (BMP180 sensor), heartrate (Pulse sensor) and ECG (ADS1292R sensor) which is widely used to collect vital signs. Sensors were connected to Raspberry Pi to send sensors data in real time to doctors. Chauhan et al. [9] mentioned that using a system that is able to provide communication between patient and doctor for sending the right data to doctor and receiving prescript from the doctor can reduce the number of visit to doctors and hospital significantly. This can clearly demonstrate that reducing patients being hospitalised can reduce costs significantly.

Predictive Analysis and Arduino as a gateway connected to sensors in patient's body to get vital data [15] is another system with the same concept which aims to detect changes in received sensory data and sends messages to operators if something goes wrong. Using the combination of ada boosting and Naive Bayes algorithm from remote sensing. Dudakiya 
et al. [15] examine the algorithm by monitoring patient's data manually for few days, and then collected to check with data gained from the sensors. In this study, Arduino is attached to the patient's body and connected to Wi-Fi for data transformation. This cannot be the best practice interim of Arduino hardware size and accuracy in kept detached to patient body and connected to the Wi-Fi. The data monitored in this work is heart rate, RR, BG and BP without mentioning the type or technologies to measure the data.

WonedRing is a wearable sensor developed to detect and analyses user's activities [46]. The sensor use for elderly people to monitor their activities in daily bases and send it to their supporters such as family member, doctor or neighbours to know information about their health status to help them to live independent safely. The system challenges to solve the issues of recognizing the activity of the user such as walking, eating, running, brushing and etc. Also, finding a useful information from monitoring user activities and deliver these data to the authorised party. User's activities are stored in databases then, the system filter these activities to get useful data and share it with supporters.

Mobile phone (Smart-phones) gateway to monitor blood pressure level via keep in touch (KIT) [14] used a close loop data transferring by connecting the mobile phone in Java to website that make all data accessible by everyone to monitor the blood pressure level. Testing blood pressure using Breakout barometric pressure sensor [14] through measuring the diastolic and systolic and report it to doctor in case of disorder detected. The normal blood pressure result is $120 / 80 \mathrm{~mm} \mathrm{Hg}$, if recorded sensory data are different than these data reported to doctor $[14,46]$.

Another example of using patient's smart-phones as the gateway communicates with a wearable sensor for healthcare monitoring architecture is presented by Chanhee et al. [22]. This system is developed to monitor patients through sensor impeded on users mobile or wearables such as heart rate sensor in smart watches. The solution for filtering the overload data (data provided by the sensor) constructed through databases querying facility. Sensor Network Query Processor (SNQP) and Sensor Network Query Language (SNQL) use to improve data acquisition for sensor network databases [26, 29]. SNQL and SNQP have different roles, the first query language support applications software advance expressiveness, while the query processor provides adequate algorithm for data accumulation from sensors.

In our research, we are using a pulse sensor connected to (Arduino Uno) to measure patient's heart-rate and send all measurements to patient's smart-phone application through a Bluetooth device. The mobile app will act as a gateway to transmit all patient's data to cloud for data processing. ${ }^{2}$ The data then will be available to access through multi-platform software such as doctor's platforms (i.e., website) to monitor and interact with patient's data and all in real-time.

\section{Proposed system}

The AAL systems, in general, aim at allowing elderly, disabled, and people with chronic conditions live safely at their home environment. In addition, AAL systems enable collaboratively assisting environment controlled by health-givers (e.g., family, friends and medical staff). These systems consist of different kinds of wearable medical sensors, smart phones, wireless sensors and actuators, computer hardware, computer networks, software

\footnotetext{
${ }^{2}$ It is worthy noting that local processing of collected data (i.e., on the Fog layer) is out of the scope of this paper.
} 
applications, which are interconnected to exchange data and provide services in an Ambient Assisted environment. The sensors and the actuators are connected with the AAL applications and patient's gateways for sending medical data to data-center, and then become available for health monitoring purposes. In this section, we will describe the network topology and framework for AAL system. However, before presenting the network topology and framework model, system architecture is given to help understanding the infrastructure of SW-SHMS.

\subsection{System architecture}

SW-SHMS make use of smart-phones and wearable sensors to monitor elderly people in real-time, thus, this proposes improving the efficiency of healthcare by providing more reliable and convenient healthcare system that enables home based monitoring. The main task for the SW-SHMS is to monitor physiological data collected from patient's wearable device, then, upon that a data record created in the data-center, and ultimately provide access to these data by authorised health-giver and doctors at anytime in anywhere. The system consists of three main layers that collaborate to achieve system goal. Each layer has its own requirements and techniques, for example, in patient's layer, the key requirements are that the sensors and the gateway should be able to communicate with the data-center to store patient's sensors data. The main layers of the system are shown in Fig. 1.

- Wearable devices (Patients Layer): A wearable device and smart-phone sensors will be connected to patient's body to collect physiological data. Such sensors are measuring vital-sign, such as, blood oxygen saturation, skin temperature and heart-rate, variety of

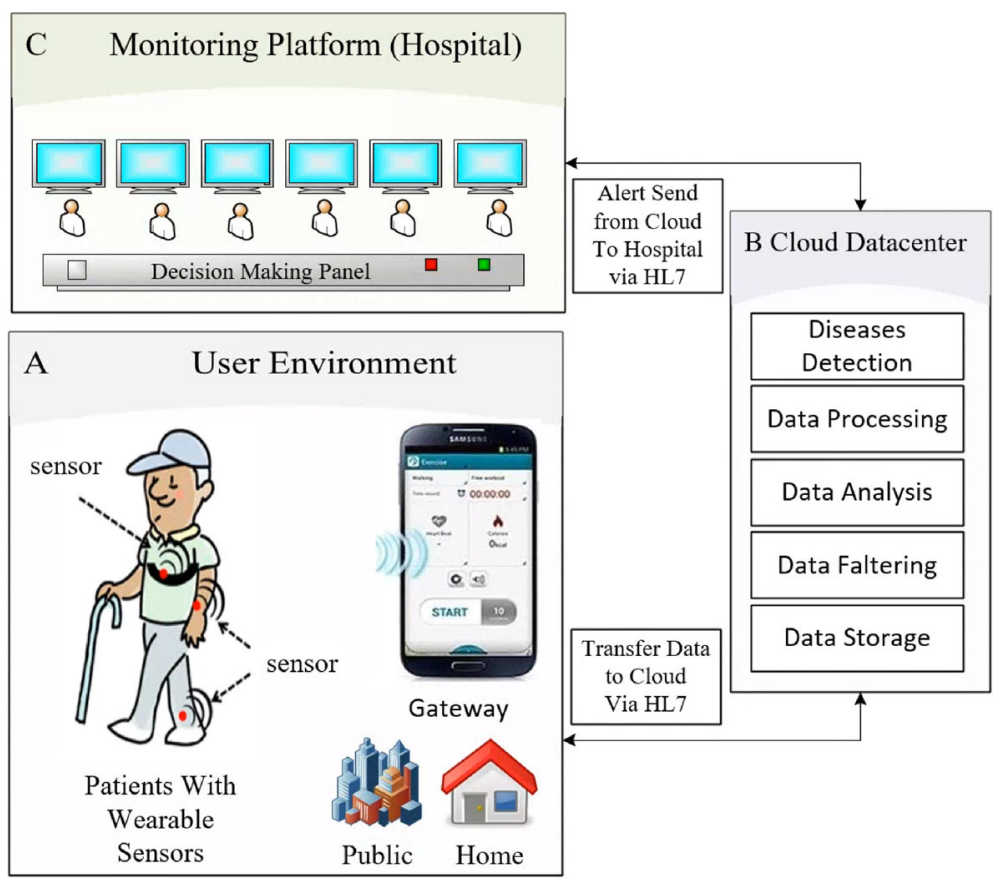

Fig. 1 High-level overview of SW-SHMS system architecture 
healthcare sensors are available today [9, 31, 34, 35, 41]. Monitoring these symptoms in patient's body is very important, as any abnormal data could potentially end up with a disease [35, 41]. For instance, drop of oxygen level in the human body causes a sleep apnea, which leads to death. In addition, abnormal blood pressure causes a kidney disease or diabetes. The sensory data are transmitted to patient's smart-phone app via Bluetooth device and ultimately to a cloud database. Moreover, sensors will operate to measure and send the data regularly without patient attention to make everything automated (i.e., IoT), thus, this enhance users quality of experience and make them more comfortable.

- Cloud (Data Layer): The Cloud refers to the place where the system data is stored and processed. Cloud receive patient's data from their smart-phone over internet to be sorted and than it become available for doctor's inspections. In addition, all data analysis and processing will be held in the cloud for any disorder detection in patient's data, thus, the abnormal changes in patient's data will be categories based on patient status and diseases. All resulted data/info will be reported either to patient's and/or doctor's platform or emergency unit or both depend on patient status. Thus, Cloud enables collaboration and knowledge sharing through its infrastructure which allows medical professionals to host information, analytics and diagnostics about patients so that other professionals around the similar interests can immediately access the data. This reveals in faster prescriptions and real-time updates to patient's data.

- Monitoring platform (Hospital Layer): This layer is a doctor's platform to monitor patient's records and sensory data. The doctors will be able to inspect reports provided by the system from the cloud and they able to take actions. Data synchronisation in this platform in real-time by pulling all data from the cloud as soon as it become ready to use to keep doctors up to date with patient's status, also to help paramedic to take early action in case of emergency before the situation getting worse and prevent hospitalisation.

\subsection{Motivating scenario \& network topology}

Improving the efficiency of healthcare and biomedical systems is one of the considerable goals of modern society. During the system design time of a healthcare system, a number of factors should be considered. However, two significant and essential design factors should be taken into consideration and related to the scope of this paper, these are: Firstly, the healthcare system should be live for 24/7 with no downtime. Therefore, a caregiver can always monitor patients conditions. Secondly, data freshness, the data should be always upto-date (aka, minimum delay). If the data is not fresh, or system is down there is a potential to cost human life. This section proposes a healthcare motivating scenario and the network topology for the proposed SW-SHMS system.

Consider an IoT healthcare system to monitor human symptoms. Based on the data for patients with chronic diseases, offered by healthcare organization call Healthcare Trust to its patients in care-homes. The system is modelled focusing on latency sensitive services and minimum down-time, therefore, the network topology shown in Fig. 2 has been developed. The main components are:

- User Environment (wearable and sensors): this represents the percipient layer, where all medical sensors and wearable are connected to patients and operated over the IoT network (i.e., able to send and receive data over this network). Each sensor/wearable 


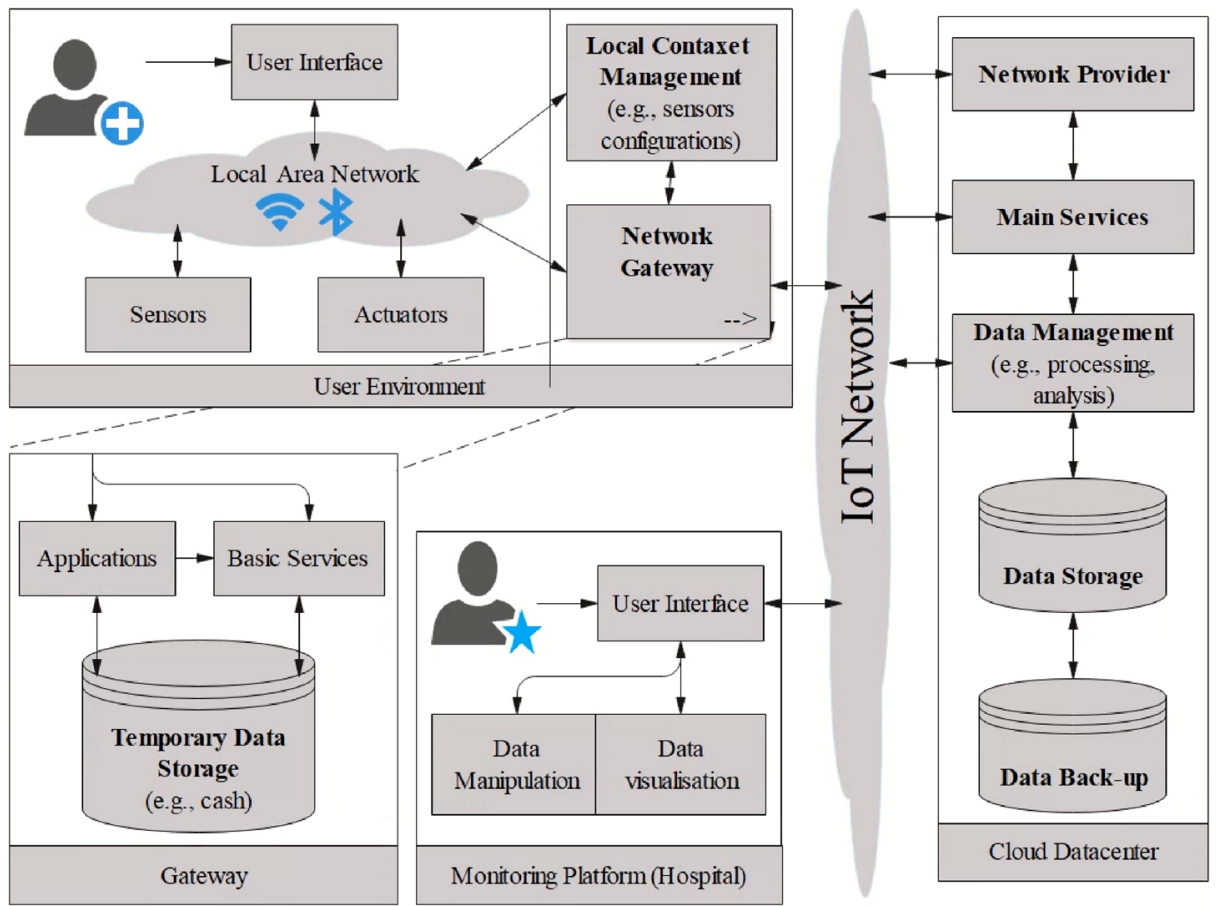

Fig. 2 WS-SHMS System Networks Model

(e.g., pulse sensor) has a unique identifier and communication capability over the network to interact with the gateway. The data gathered within this layer can be biomedical data (directly from patient's body) and/or environmental data (from the surrounding). The generated/gathered data in this layer are transmitted to the gateway via wireless or wired communication protocols, such as Bluetooth, ZigBee, and Wi-Fi.

- Gateway: this part is responsible for interacting with patients sensors which are used to detect patient's symptoms and perform prime processing on the sensed data. Outcome of this segment is a summary of patients conditions sent to the caregivers. In addition, the Gateway is also able to react upon symptoms abnormality when it's been detected, for instance, sending a help-request (e.g., request for caregiver for assistant) or emergency-request (e.g., call for ambulance) as soon as a emergency situation is detected.

- Cloud Data-center: this segment was deployed to act on data provided from the Gateway. Thus, the main data storage and disease detection activity are executed. This includes the machine learning (ML) activities for data training and analysis to detect diseases and abnormality.

- Monitoring Platforms: this includes both patient's platform and caregivers platform. Thus, each dashboard provided for data visualisation, monitoring, and functionality controls over the collected data from sensors. This platform requires the level of security access alongside with different access layers, for example, patients users can have the master level of access, while caregiver have restricted access according to the granted level of access. 
The other components of the adopted network topology are used primarily to manage the network configuration settings and interactions among system platforms and its entities. Moreover, in the user environment, the local context management is available within the network to setup and manages sensor's related functions such as active/inactive timestamps and frequency of sensing. Thus, the local context management interact with both local area network for deploying sensors configuration settings and updates, and the gateway to inform/update the services that are running within the network about the new changes. Similarly, within the could data-centre the data management component is existing to manage the data manipulation processes, such as data analysis, according to the services provided.

With the view of Healthcare Trust to deploy the system at their care-home premises, the system infrastructure would include the segments mentioned beforehand. The Heal thcare Trust IT department has to install a number of Gateways on each carehome (depending on the care-home size) with a minimum of two nodes (Gateway, and Gateway $y_{2}$, alongside a detected cloud data-centre (cloud 1 ) for ML Engine. Ultimately, provides the health-giver with access authentication to the Monitoring Platform dashboard. IT division experts, justify the purpose of deploying the system with at least two Gateways at any given location is to make sure that a backup node is available in the case of one Gateway going down. In addition, in the case of Gateway $y_{1}$ being loaded,

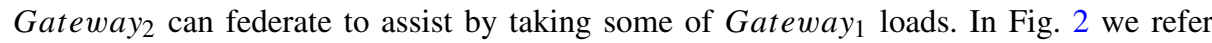
to the intercommunication network as IoT network is differ from the traditional network by contents and/or servers generations and consumption. Thus, the IoT network is more interactive. Moreover, within the traditional network, the contents and/or servers are generated or consumed by humans through manual requesting for a service or data acquisition, while in the IoT network the contents and/or servers are generated or activated mostly through a things (i.e., sensors and embedded systems) (Fig. 3).

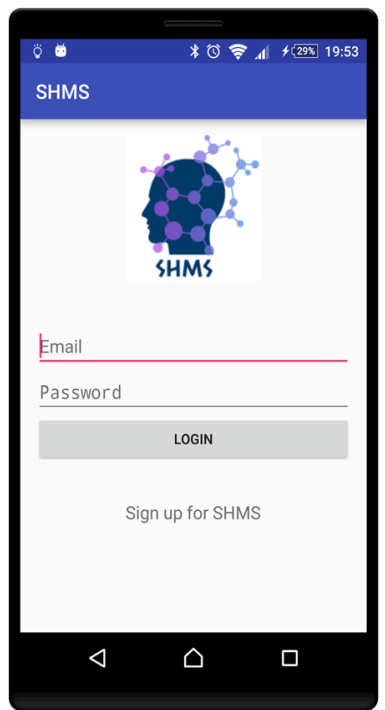

(a) Login Page

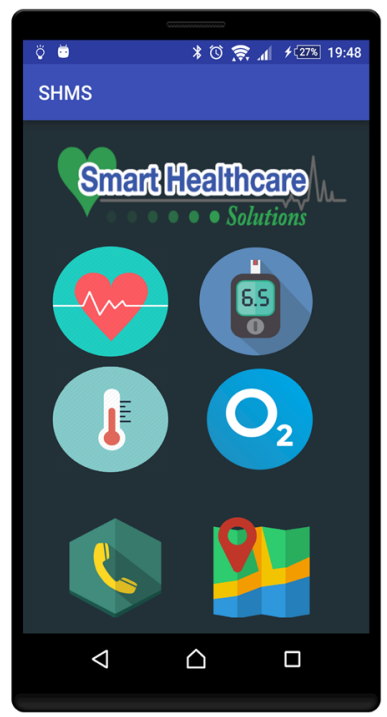

(b) Home Screen

Fig. 3 Patient's platform 


\section{System requirements and implementation}

In this section we highlight the main components for developing the AAL system (i.e., SWSHMS), and implementing a prototype for the proposed system, which aims at monitoring elderly people vital sign at their home and/or work. SW-SHMS monitor patient's physiological data (e.g. heart-rate). All collected data will be transferred from sensors to Gateway and Cloud to be processed to check any disorder or abnormality within the collected data. Reports will be generated for the processing activity and it will send to patient's doctor and other parties that provide healthcare (e.g., hospital) with respect to the privacy and authentication of the data. Algorithm 1 highlight the interactions among system components. The main task for the system is to monitor physiological data collected from patient's wearable device as per lines 4-7, than, sensed data will be recorded will be send to be stored in the data-center as per lines 8-12, and ultimately provide access to these data by authorised health-giver and doctors at any-time in any-where via doctors dashboard as per lines 11 and 12.

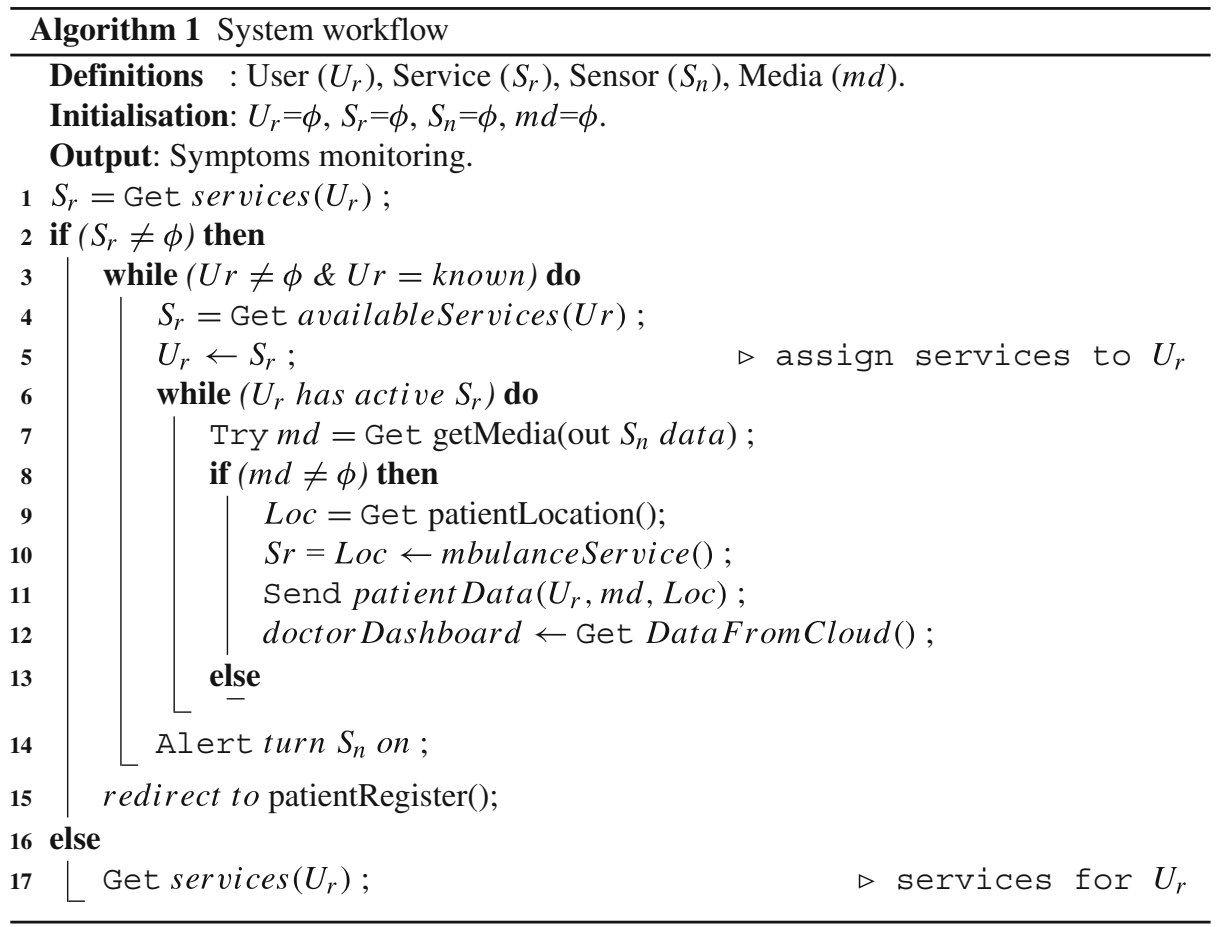

\subsection{System requirements}

Healthcare solutions are carefully designed because it is crucial and involve dealing with patient's life. Thus, developing an effective healthcare monitoring system, several factors need to considered, such as data availability, consistency, and reliability. However, the most important factor is data freshness that needs to be well supported during runtime [20, 31] by the healthcare systems which deal with people health. More precisely, when authorised people monitoring patient's symptom, the data it has to be refreshed with high frequency so that doctor's platform update continuously to pull the latest data from patients with warbles. 
Healthcare systems rely on several approaches to transfer patient's data from one platform to another. Some of these approaches can be internal, like data is already on one of the system platforms then it sends the data in a secure channel to other platforms. While other approaches require middle-ware devices to collect these data and send it to system platforms, this what so-called system Getaway [15]. The basic gateways performance is to accumulate data from sensors or any wearable devices that measure patient's physiological data, then send it to data-centres (e.g., Cloud data-centre). Thus, in this instance gateway can be seen as a bridging point, for example, the UT-GATE [37], is provide data monitoring from original data sources (e.g., sensors) to the main monitoring platform. Moreover, there are different type of devices that can be used as a gateway such as a network router, and smart-phones $[15,20,34,35]$. The Gateway, can be any micro-controller that is programmed to act on the data able to transmit from one platform to another.

Wireless sensor network (WSN) is the core of smart healthcare monitoring systems, where it represents than main sources of data, thus providing symptom physiological data [8, $9,41]$ from patient's body. There are different type of sensor use in healthcare monitoring, each sensor has different capability [34], for instance, Pulse sensor use to measure heartrate [34], BMP180 sensor measuring blood pressure [9], and ADS1292R sensor use for both ECG and respiration [9, 34]. These sensors provide data from patient's body in real-time, and the output data is either in analogue format or digital format. Therefore, the sensors should be connected to an appropriate Gateway that is able to read sensors output data and act on it or transmitted to data-centre.

In this research, Arduino Uno and patient's smart-phone will be used as gateway to prove the concept of the proposed solution. However, healthcare solutions must have elasticity design, to be adapted with growth of the system, and hardware components must be improved continuously, due to the fact that hardware components are enhanced rabidly these days.

\subsection{Implementation}

The development of the proposed system starts with a database that is able to handle system services and storing patient data. The entity relationship diagram of the data based is depicted in Fig. 4. The database comprises 11 tables that have been constructed to handle system data interactions and functionalities. The tables and attributes have been selected carefully to avoid any overlapping, dependencies and data redundancy that could burden the network and slow down the performance of the system. The process of storing and retrieving data within the system have two-part processes. First process involves data encryption/decryption according to the appropriate standard of patient's data security. Second process involves the method of data representation changes (i.e., for storing data). For example, analogue data outputs from sensors are converted to some readable row-data format to be stored in the data repositories. Thus, stored data can be retrieved by another process at a later stage and the same method of data representation will be applied to provide a proper data visualisation. Moreover, the development of system platforms starts with implantation of patent's platform of the SW-SHMS. Patient's platform is consists of a mobile application that able to communicate with the sensors to accumulate patient's vital sign. Android studio version 2.5 has been used to implement the mobile application. Figure 3 shows the login page and the main screen of the SW-SHMS application.

A pulse sensor has been used to measure patient's heart-rate and transfer the data through Bluetooth device to the mobile app. The pulse sensor used to measure heart-rate through an optical heart-rate chip. The output data will be analogy data format. Therefore, Arduino 


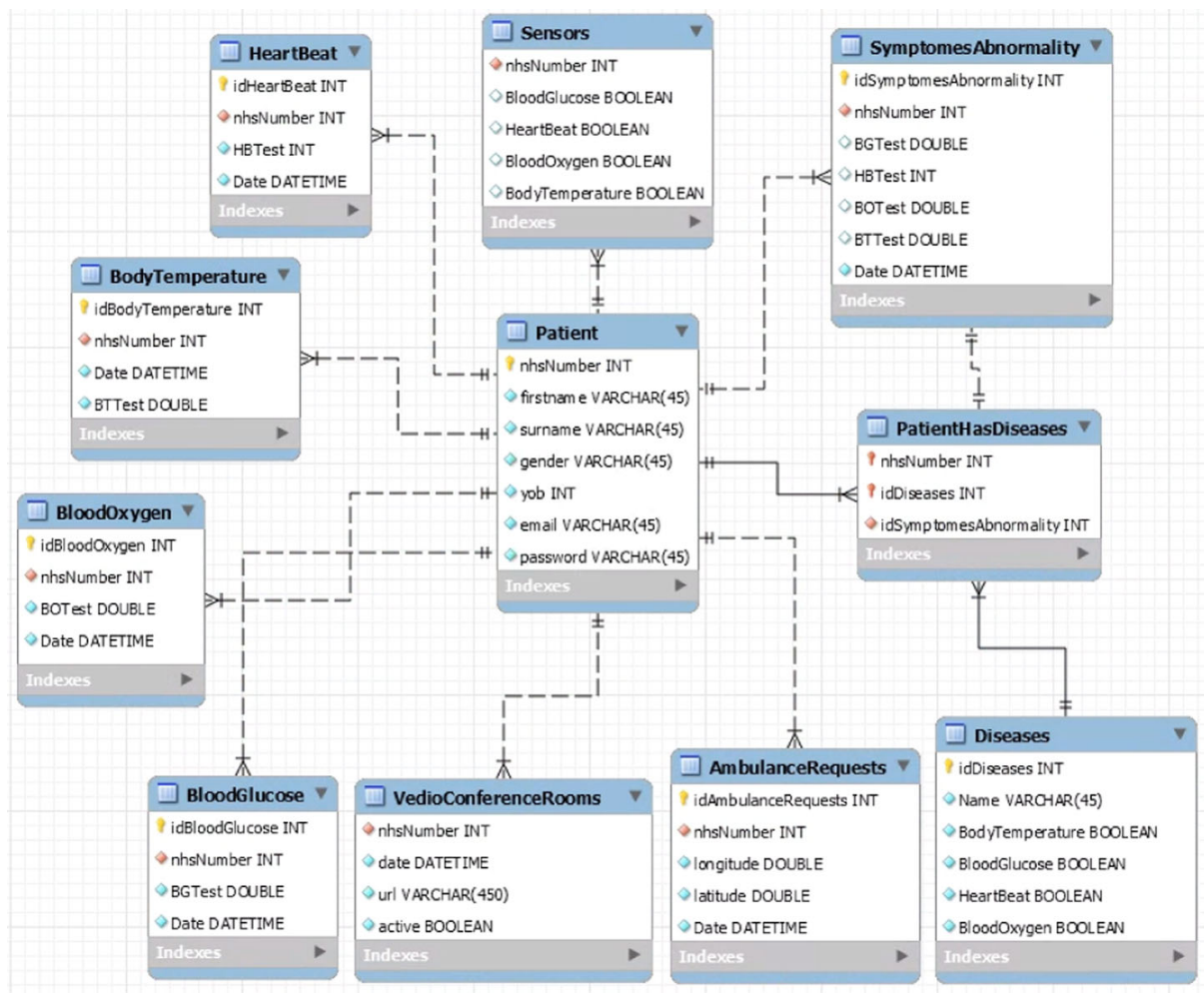

Fig. 4 System Database Design

UNO has been used to receive the pulse sensor readings. The reading of the sensor sends to patient mobile app through the HC-06 Bluetooth device which is connected to the Arduino UNO. Figure 5 shows the connection between the pulse sensor and the Arduino UNO. The pulse sensor has three points of connections as shown in Fig. 5. The first one, is the red coloured wire which is the power wire, this wire accept voltage value between $+3 \mathrm{~V}$ to $+5 \mathrm{~V}$. The black wire represents the ground connection (GND). The blue wire is responsible for transferring the signal from the sensor to the Arduino UNO. The open-source Arduino Software has been used to program the UNO in order to receive and send sensor readings.

The following step involves sending the sensory data to the SW-SHMS mobile app. After sensors' readings have been collected from the Pulse sensor in the Arduino UNO, it will be sent to the android app through a Bluetooth device. Therefore, the UNO has been connected with a Bluetooth device, which is the HC-06 version. The physical connection between the Bluetooth HC-06 device and the Arduino UNO board is shown in Fig. 6. Arduino Bluetooth version HC-06 has four connection points, these are VCC, GND, TXD and RXD. The four connection points need to be connected to the UNO board in order to complete the operation of sending Pulse readings to the app through the HC-06. Moreover, the Bluetooth device connected to the Arduino board through the following wires: 1) Connecting Bluetooth VCC pin to $5 \mathrm{~V}$ pin on the Arduino board. 2) Connecting Bluetooth GND pin to the GND pin on the Arduino board. 3) Connecting Bluetooth TXD pin to RXD pin on the Arduino board. 4) Connecting Bluetooth RXD pin to TXD pin on the Arduino board. 


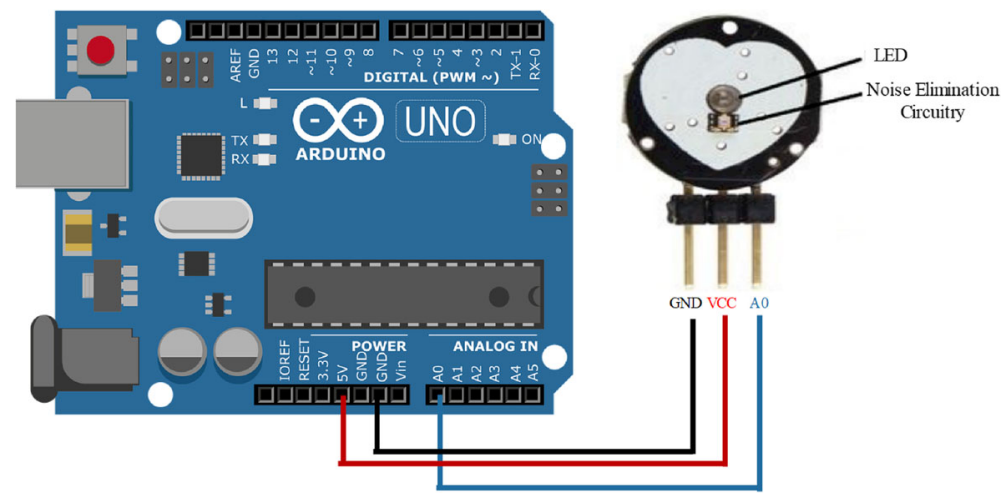

Fig. 5 Pulse sensor wires connections to the Arduino UNO

In order to send sensor data from the Arduino to SW-SHMS mobile app through the HC-06, it is required to print these data in the Arduino series after configuring the correct serial use by the Arduino board. Then, the Bluetooth device will be able to read these data from the UNO serial and send it to the connected device. For every Bluetooth device there is a mac address use during the connection between the mobile apps (using smartphone Bluetooth device) and Arduino Bluetooth device, the mac address for the used device is [20:16:05:03:06:84]. One of the main functionalities of SW-SHMS is to monitor heartrate and other symptoms therefore users will be able to monitor these sensors data on their mobile app. The result of sensors readings are sent to patient smart-phone through the Bluetooth device, however the application must receive these data and display it on the monitor screen inside the app. This app will be responsible for making the connection to the Arduino Bluetooth device through a media access control address (MAC) via the universal unique identifier (UUID). This can be generated for each device or just using a stander identifier configure for the app. Once the connection made, data will be displayed on SW-SHMS patient's app as per Fig. 7. In addition, they will have the ability to send these data to the Cloud, where all patient's records are kept.

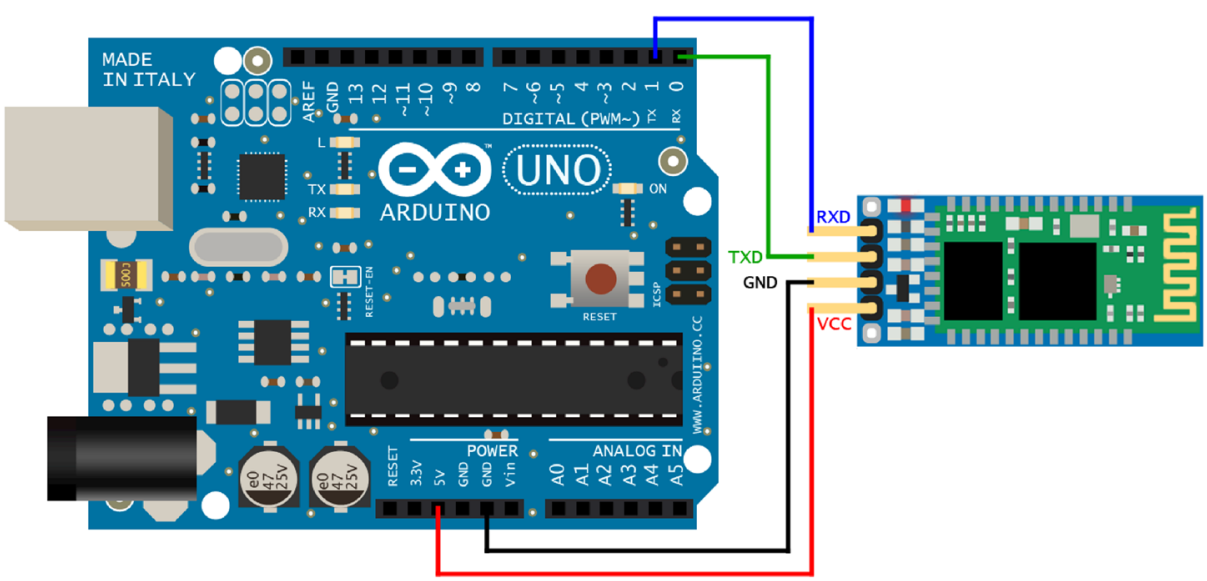

Fig. 6 Bluetooth HC-06 wires connections to the Arduino UNO 


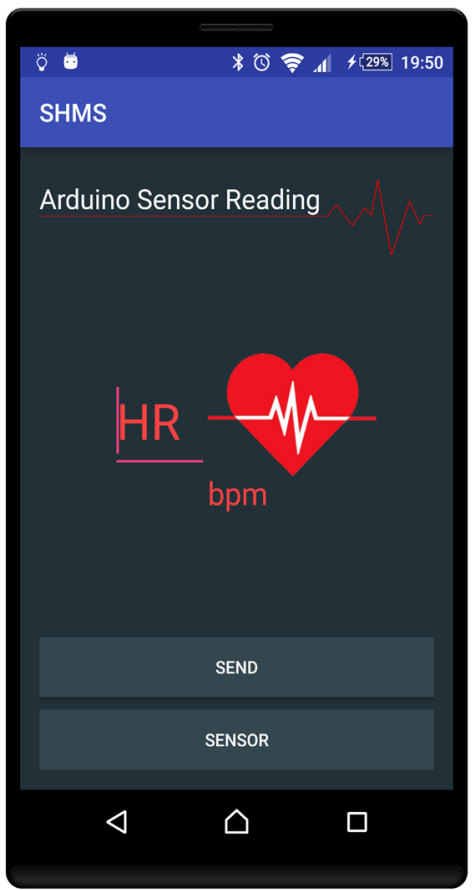

(a) Readings from the sensor

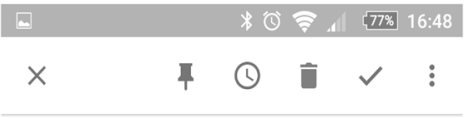

\section{Warning! Heart Rate}

NHS

Dear Patient Your Heart Rate is abnormal,...

(N) $\begin{aligned} & \text { NHS } \\ & \text { to me }\end{aligned}$ 16:44:

Dear Patient

Your Heart Rate is abnormal, please contact your doctor for more details

Regards,

NHS Team

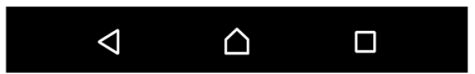

(b) Email Notification

Fig. 7 Heart-rate monitor

The development of SW-SHMS includes data tracking for data disorder. For instance, the normal heart-rate for an adult is between 60 to 100 beats per second. Therefore, any data received by the system out of this range should be detected. Moreover, once data has been received in SW-SHMS data-centre, it will be stored accordingly in data categories. Patient's information such as name and gender will be stored directly in the system. While symptom data such as heart-rate, and blood oxygen will be examined first to check if any of these data are abnormal, which mean it is exceeding the normal or standard values. The standard data of the symptoms has been taken from symptom-checker tool in patient.info. ${ }^{3}$ To achieve this action number triggers developed to track the inserted data to the data-centre, when abnormal data detected at any-time it will be recorded and push these records to patient's doctor, also, patient will receive an email to inform them about symptom disorder as per Fig. 7b. The full development environment where all system patient's platform components are connected (i.e., Pulse sensor, the HC-06 Bluetooth device, and the Arduino board are all connected together) shown in Fig. 8.

The hospital or doctor's platform of the SW-SHMS system is a web base application to interact with patient's platform. The requirements of this platform is to show results and information from patient's app such as symptom data and diseases. One of the important functions for the hospital and/or doctor platform is to provide a list of patients who is waiting for treatments, this list will be appear on doctor's dashboard as per Fig. 9. Patients list is

\footnotetext{
${ }^{3}$ patient.info (https://patient.info/symptom-checker) is a registered website in England and Wales use in most
} GPs. 
Fig. 8 Full development environment for SW-SHMS patient's platform

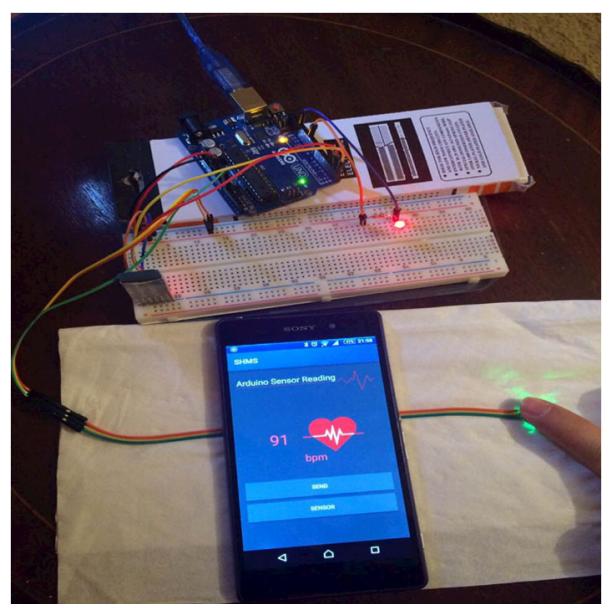

created upon disorder founded in patient's records. The symptoms data plotted on doctor's platform from the Cloud using a line chart that shows the time and value of sensor reading as per Fig. 9b. The symptom's monitor refreshs periodicity to get real-time updated data of symptoms.

\section{Results \& evaluation}

SW-SHMS deals with patient's data directly, therefore, system scalability and latency must be well examined to make sure that the system can achieve high performance in dealing with elderly people data and effectively monitor their health in real-time. In addition, system scalability is that the system should be fixable to expand accordingly with the growing number of the population and patients using the system. In this section, we highlight some results and evaluations obtained from the developed prototype in term of efficiency and scalability.

In SW-SHMS, the latency is dependent on two main factors. On one hand, hardware components, which involve the response time between the Bluetooth device and the gateway, as well as, the network speed to deliver data from the gateway to Cloud. On the other hand, the software system that performs the data transformation and processing, which involves compressing data while sending and process accumulated data from sensors through each transaction. Moreover, the process of detection symptoms disorder in the running prototype seems to be the longest operation, as it is involve collecting data, detecting symptom disorder through processing the data and compare it to normal values, then report it to specialists and email patient with the detected situation in case of disorder. We have carried an experiment using the developed prototype to simulate the scenario in Section 3.2 where data from patients are accumulated and processed by the SW-SHMS system for 24 hours, thus the overall system response time is promising, as per Fig. 10a the response time for most requests is less than $1 \mathrm{~ms}$ during the simulation of $1 \mathrm{k}, 2 \mathrm{k}$ and $3 \mathrm{k}$ requests. Figure $10 \mathrm{~b}$ show the number of packets that are sent by the system along with the number of packets received and dropped in the network during the execution time. In addition, Fig. 10c demonstrates the total end-to-end latency for streaming data continuously over a 24 hours period. The 


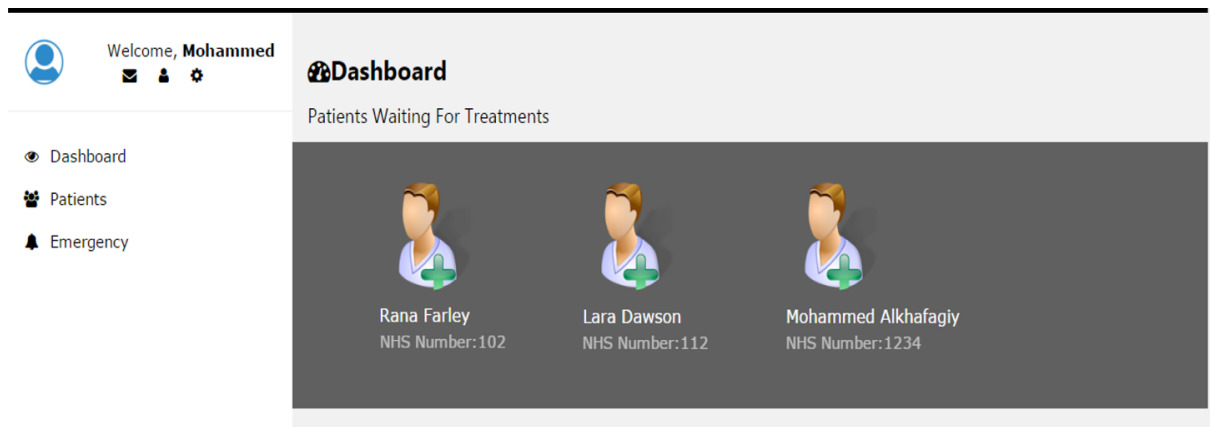

(a) Dashboard

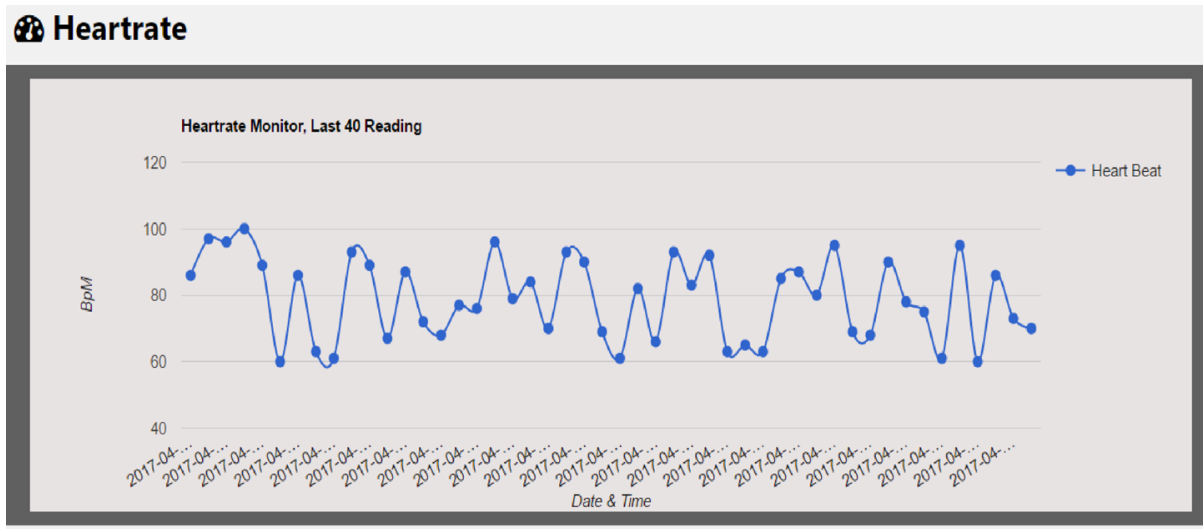

(b) Symptom monitor

Fig. 9 SW-SHMS Doctor's platform

configuration set for date to travel from Sensor $\longmapsto$ Gateway $\longmapsto$ Cloud, and than data processed and become available to doctor's platform.

The results in Fig. 10 are promising, thus, the total number of sent packets is around 3500 packets, 3432 packets are received and only 68 are dropped by the network. The overall dropping ratio of all sent packets is around or less than $2.2 \%$ which is promising ratio and way lass than the acceptable threshold of $15 \%$ as everything above that rate noticeably deteriorates the availability of the network [42]. Moreover, Fig. 10c show the total latency in millisecond (ms) for transmitted packets, thus, must packets are received with ( $0 \mathrm{~ms})$ latency, however, the longer latency found where $(6 \mathrm{~ms})$ for less than $4 \%$ of the packets, out of the overall number of 5000 packets.

Likewise, the scalability factor of the system is a big aspect as it effect the performance of the system significantly. This factor is measured based on the quantity of data need to be processed over the Cloud. In future Internet architecture, there is a big concerns about the amount of data that is stored in the Internet as it expected to be huge in which cause a latency and unreliability to network. Therefore, in our proposed system the data is filtered by the gateway before it has been send to the cloud, thus, in this case we will only keep data that are unique and valuable by removing all redundant data via the gateway filtering process. In SW-SHMS the main data source is patient's sensors, hence, all factors of data size and 


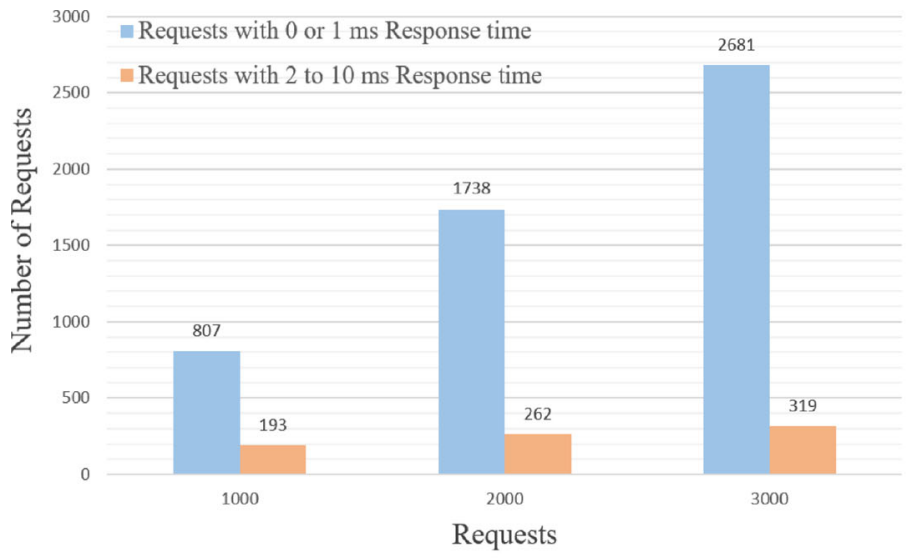

(a) Requests Response time

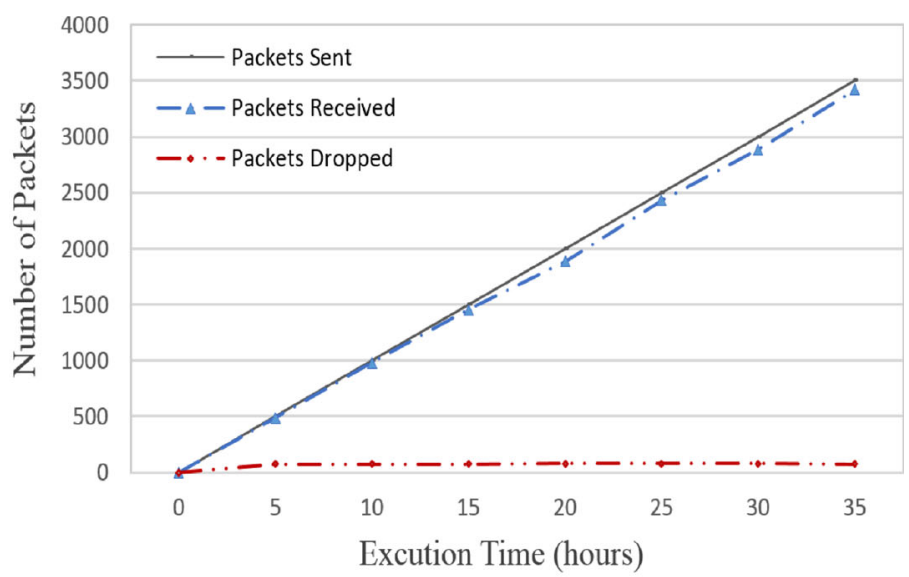

(b) Packets Sent, Received and Dropped

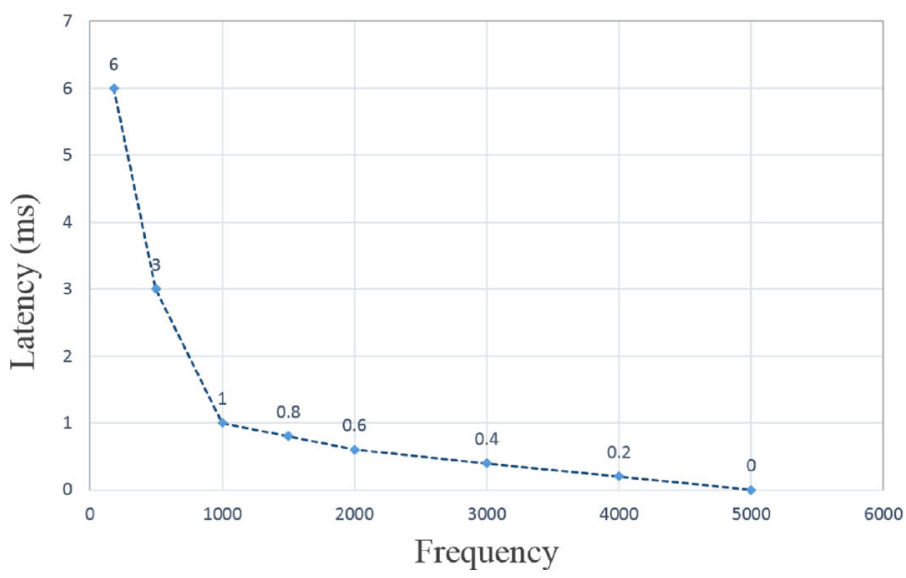

(c) Number of messages per latency

Fig. 10 SW-SHMS packet's transformation and latency 
frequency are relies on the pre-set rules by the Getaway. For example, the amounts of data specified for patient's sensor can be set to one record per hour per sensor unless the record unique or required by other services. Theoretically, if we considering that the data update rate is two packet per hour (unique packet), and we are using four sensors (e.g., heart-rate, blood oxygen, blood pressure and body temperature) for the duration of 24 hours, this means we have $2 * 24=48$ packet per sensor. Then, if we multiply this number by the number of connected sensors, we will get only 192 packets a day from out four sensors. Based on this, we have measured the amount of storage that this data can consume using our prototype, hence, the result for one sensor is approximately 32 byte worth of data per person (i.e. the total storage for four sensors is 128 byte per day). Figure 11 gives indication about the amount of data can be gained from 500, 1000, 1500 and 2000 connected patients with four sensors for each patient. In general, these results in Fig. 11 is subject to changes according to the number of connected patients on the system as well as the number of sensors required for each patient and the duration of monitoring (i.e, frequency of data).

There is a high flexibility in the SW-SHMS system to grow with the increasing numbers of sensors and patients connected to the system. SW-SHMS can be expanded simply by adding more gateways that are capable for handling patient's sensor data. Also, according to the workload such as data traffic several gateways can be added to collaborate with each other to handle the load, thus, enhancing system reliability and performance.

In term of system costs, SW-SHMS similar to other systems that includes both hardware and software in the developments process, hence, it relies on several cost factors, which can significantly increase or decrease the expenses of the system (such as the Gateway capacity and quality). The main cost factors are the hardware components quality and software functionalities, which are the main component of each platform in the system. The total price of system installation will depend on the following: i) system size, this includes addeda number of Gateway, sensors, data-centres alongside with sensor types, quality and quantity. ii) number of platforms in the system. iii) software functionalities, performance, and security aspects. However, installation cost is a one time cost which is normal for any new system and is subjects to changes upon system requirements. Thus, the important costs are maintenance and data costs, the cost for data management and storage are important in the long term. Therefore we estimated the data costs for the running prototype according to Google Cloud

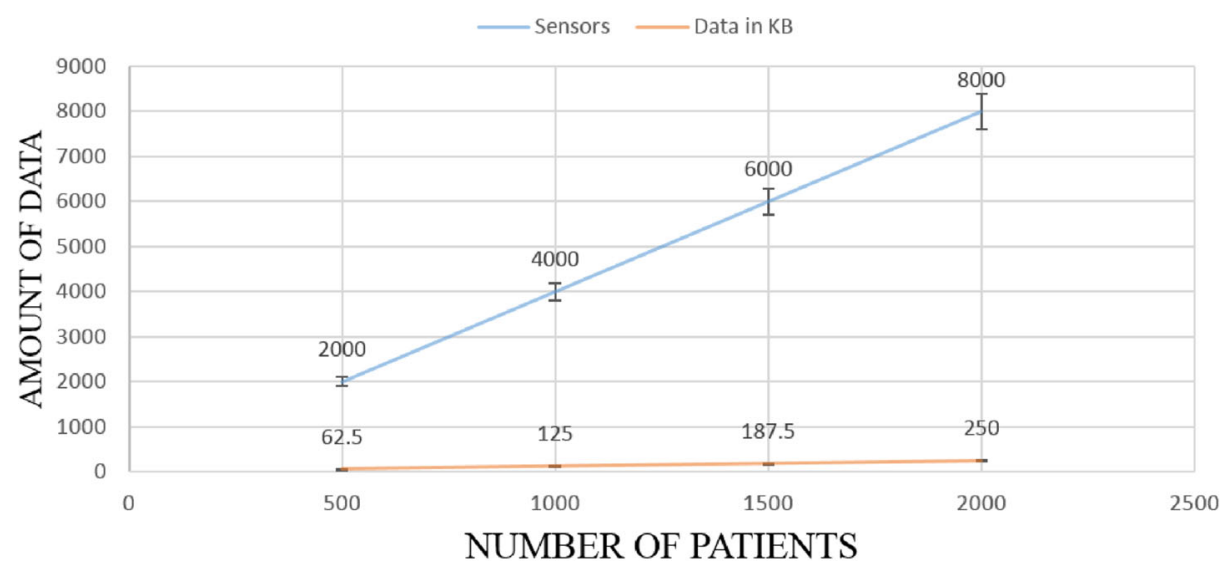

Fig. 11 SW-SHMS patient's data scalability 


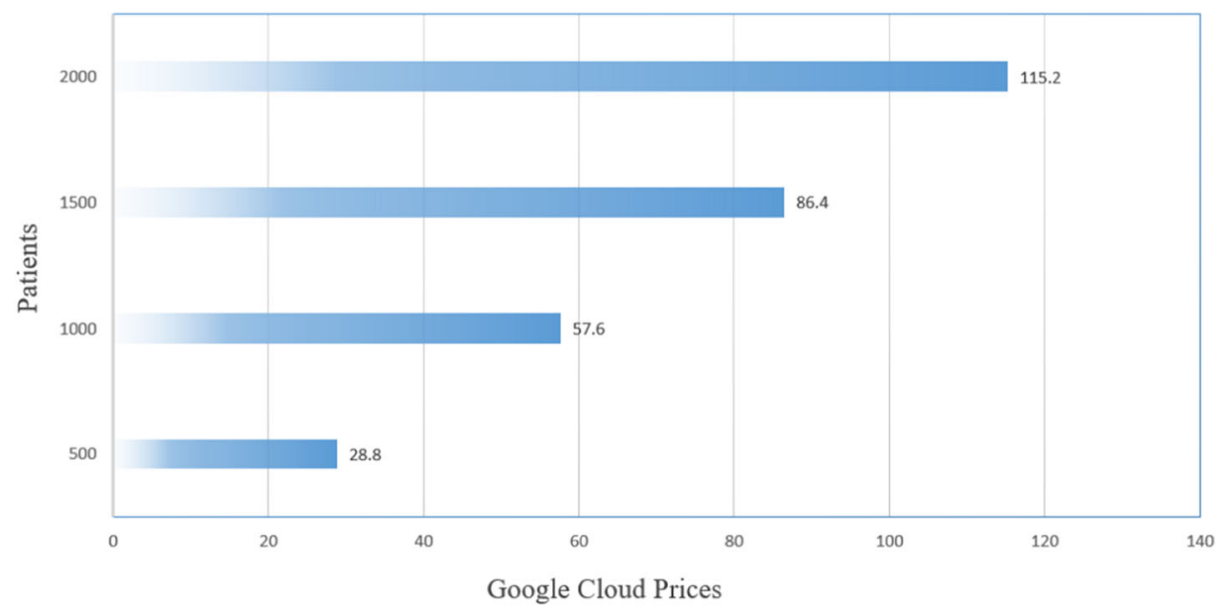

Fig. 12 SW-SHMS patient's data costs according to Google cloud pricing

prices. ${ }^{4}$ In order to estimate the expenses of data managements, we have used the data storage size calculated before (i.e., 32 Bytes) alongside with estimated number of read, write, and process for each sensor. From the running prototype, the total data size for one patient per day is about $0.128 \mathrm{~KB}$. Figure 12 give an estimate prices, based on 500, 1000, 1500 and 2000 connected patients and the Google could pricing for data storage and processing per month.

\section{Conclusion \& future work}

The smart healthcare monitoring system (i.e., SW-SHMS) has been proposed in this paper to handle the challenges of providing home based healthcare monitoring and avoiding hospitalisation. The literature show that there is a great demand of producing an effective healthcare solution that monitor elderly people in their home and in real-time. SW-SHMS can highly contribute to provide comfortable, and safe environment for elder and disable people, thus, enable them to live independently without the fear of any emergency or critical healthcare situation through continuous monitoring of their health by SW-SHMS. Briefly, SW-SHMS accumulate patient's physiological data via wearable sensors and transmit it to Cloud for data analysing and processing. Thus, any detection of disorder in patient's data will be reported to patient's doctors via hospital platform. SW-SHMS has a fixable architecture that can scale and expand easily, thus, providing a reliable and cost effective systems to monitor patients remotely. In addition, the results shows that the system could efficiently contribute to improve healthcare services by using the prosed impeccable SW-SHMS system which able to monitor patients symptoms remotely and in real-time.

Future developments and enhancements for the SW-SHMS are wide, for instance, the system can be extended to apply artificial intelligence and/or machine learning concepts to help predicting life threatening diseases early. Moreover, the system holds a fair amount of medical data which be invested to develop a recommendations system that able to give a recommendations about the health and diets for better life style. In addition, the network

\footnotetext{
${ }^{4}$ Google Cloud platform. Available at https://cloud.google.com/storage/pricing.
} 
topology can expand to have Fog computing node which will help processing the data at the edge of the network instead of traveling for long distance to Cloud, also, Fog computing cloud reduce data traffic on the cloud and help to make better healthcare decisions due to it's location within the local area network. Thus, Fog computing will be employed to reduce the packets loses by upgrading the network infrastructure to allow distributing several Fog nodes at the edge of the network. This will help in empowering the system in data acquisition and manipulation via Fog-Cloud collaboration model that act faster on the data packets before it gets lost.

Open Access This article is distributed under the terms of the Creative Commons Attribution 4.0 International License (http://creativecommons.org/licenses/by/4.0/), which permits unrestricted use, distribution, and reproduction in any medium, provided you give appropriate credit to the original author(s) and the source, provide a link to the Creative Commons license, and indicate if changes were made.

Publisher's note Springer Nature remains neutral with regard to jurisdictional claims in published maps and institutional affiliations.

\section{References}

1. Adlam T, Faulkner R, Orpwood R, Jones K, Macijauskiene J, Budraitiene A (2004) The installation and support of internationally distributed equipment for people with dementia. IEEE Transactions on Information Technology in Biomedicine

2. Ali A, Ming Y, Chakraborty S, Iram S (2017) A comprehensive survey on real-time applications of WSN. Fut Internet 9(4):77

3. Alsina-Pagès RM, Navarro J, Alías F, Hervás M (2017) homeSound: Real-Time Audio Event Detection Based on High Performance Computing for Behaviour and Surveillance Remote Monitoring. Sensors

4. Angelini L, Carrino S, Abou Khaled O, Riva-Mossman S, Mugellini E (2016) Senior living lab: an ecological approach to foster social innovation in an ageing society. Fut Internet 8(4):50

5. Barnes NM, Edwards NH, Rose DA, Garner P (1998) Lifestyle monitoring-technology for supported independence. Computing \& Control Engineering Journal

6. Bonato P (2010) Wearable sensors and systems. IEEE Eng Med Biol Mag 29(3):25-36

7. Bouchard B, Giroux S, Bouzouane A (2007) A keyhole plan recognition model for Alzheimer's patients: first results, vol 21

8. Catarinucci L, De Donno D, Mainetti L, Palano L, Patrono L, Stefanizzi ML, Tarricone L (2015) An IoT-aware architecture for smart healthcare systems. IEEE Internet Things J 2(6):515-26

9. Chauhan J, Bojewar S (2016) Sensor networks based healthcare monitoring system. In: International conference on inventive computation technologies (ICICT). IEEE, Vol 2, pp 1-6

10. Chen TL, King CH, Thomaz AL, Kemp CC (2011) Touched by a robot: an investigation of subjective responses to robot-initiated touch. In: 2011 6th ACM/IEEE international conference human-robot interaction (HRI)

11. Chouvarda I, Antony R, Torabi A, Weston J, Caffarel J, van Gils M, Cleland J, Maglaveras N (2013) Temporal Variation in telemonitoring data: on the Effect of Medication and Lifestyle Compliance. International Journal of Bioelectromagnetism

12. Cook D, Das SK (2004) Smart environments: technology, protocols and applications. Wiley, New York

13. Dall TM, Gallo PD, Chakrabarti R, West T, Semilla AP, Storm MV (2013) An aging population and growing disease burden will require alarge and specialized health care workforce by 2025 . Health affairs

14. Dohr A, Modre-Opsrian R, Drobics M, Hayn D, Schreier G (2010) The internet of things for ambient assisted living. In: 2010 seventh international conference on information technology: new generations (ITNG), pp 804-809

15. Dudakiya S, Galani H, Shaikh A, Thanki D, Late RA, Pawar SE (2016) Monitoring mobile patients using predictive analysis by data from wearable sensors. In: International conference on electrical, electronics, and optimization techniques (ICEEOT). IEEE, pp 332-335)

16. El-Darzi E, Vasilakis C, Chaussalet T, Millard PH (1998) A simulation modelling approach to evaluating length of stay, occupancy, emptiness and bed blocking in a hospital geriatric department. Health Care Manag Sci 1(2): 143 
17. Gupta MS, Patchava V, Menezes V (2015) Healthcare based on IoT using Raspberry Pi. In: 2015 international conference on green computing and internet of things (ICGCIoT). IEEE, pp 796-799)

18. Hassanalieragh M, Page A, Soyata T, Sharma G, Aktas M, Mateos G, Kantarci B, Andreescu S (2015) Health monitoring and management using Internet-of-Things (IoT) sensing with cloud-based processing: opportunities and challenges. In: 2015 IEEE international conference on services computing (SCC). IEEE, pp 285-292)

19. Islam SR, Kwak D, Kabir MH, Hossain M, Kwak KS (2015) The internet of things for health care: a comprehensive survey. IEEE Access 3:678-708

20. Kumar R, Rajasekaran MP (2016) An IoT based patient monitoring system using raspberry Pi. In: International conference on computing technologies and intelligent data engineering (ICCTIDE). IEEE, pp $1-4$

21. LeBellego G, Noury N, Virone G, Mousseau M, Demongeot J (2006) A model for the measurement of patient activity in a hospital suite. IEEE Transactions on information technology in biomedicine

22. Lee C, Kim T, Hyun SJ (2016) A data acquisition architecture for healthcare services in mobile sensor networks. In: 2016 International conference on big data and smart computing (BigComp). IEEE, pp 439-442

23. Lin CT, Ko LW, Chang MH, Duann JR, Chen JY, Su TP, Jung TP (2010) Review of wireless and wearable electroencephalogram systems and brain-computer interfaces-a mini-review. Gerontology 56(1):112-9

24. Luprano J, De Carvalho P, Eilebrecht B, Kortelainen J, Muehlsteff J, Sipila A, Solà J, Teichmann D, Ulbrich M (2013) HeartCycle: advanced sensors for telehealth applications. In: 2013 35th annual international conference of the IEEE engineering in medicine and biology society (EMBC)

25. Luprano J, Solà J, Dasen S, Koller JM, Chételat O (2016) Combination of body sensor networks and on-body signal processing algorithms: the practical case of MyHeart project. In: null. IEEE, pp 76-79

26. Madden SR, Franklin MJ, Hellerstein JM, Hong W (2005) TinyDB: an acquisitional query processing system for sensor networks. ACM Trans Database Syst (TODS) 30(1):122-73

27. Manzano-Santaella A (2010) From bed-blocking to delayed discharges: precursors and interpretations of a contested concept Health services management research

28. Mégret R, Dovgalecs V, Wannous H, Karaman S, Benois-Pineau J, El Khoury E, Pinquier J, Joly P, André-Obrecht R, Gaëstel Y, Dartigues JF (2010) The IMMED project: wearable video monitoring of people with age dementia. In: Proceedings of the 18th ACM international conference on multimedia

29. Mottola L, Picco GP (2011) Programming wireless sensor networks: fundamental concepts and state of the art. ACM Comput Surv (CSUR) 43(3):19

30. Navarro J, Vidaña-Vila E, Alsina-Pagès RM, Hervás M (2018) Real-Time Distributed architecture for remote acoustic elderly monitoring in Residential-Scale ambient assisted living scenarios. Sensors (Basel Switzerland) 18(8):1

31. Nienhold D, Dornberger R, Korkut S (2016) Sensor-Based Tracking and Big Data Processing of Patient Activities in Ambient Assisted Living. In: 2016 IEEE international conference on healthcare informatics (ICHI). IEEE, pp 473-482

32. Oresko JJ, Jin Z, Cheng J, Huang S, Sun Y, Duschl H, Cheng AC (2010) A wearable smartphone-based platform for real-time cardiovascular disease detection via electrocardiogram processing. IEEE Trans Inf Technol Biomed 14(3):734-40

33. Palumbo F, Ullberg J, timec A, Furfari F, Karlsson L, Coradeschi S (2014) Sensor network infrastructure for a home care monitoring system. Sensors

34. Pantelopoulos A, Bourbakis NG (2010) A survey on wearable sensor-based systems for health monitoring and prognosis. IEEE Trans Syst Man Cybern Part C (Appl Rev) 40(1):1-2

35. Patel S, Park H, Bonato P, Chan L, Rodgers M (2012) A review of wearable sensors and systems with application in rehabilitation. J Neuroeng Rehabil 9(1):21

36. Pham M, Mengistu Y, Do HM, Sheng W (2016) Cloud-Based Smart Home Environment (CoSHE) for home healthcare. In: 2016 IEEE international conference on automation science and engineering (CASE). IEEE, pp 483-488

37. Rahmani AM, Thanigaivelan NK, Gia TN, Granados J, Negash B, Liljeberg P, Tenhunen H (2015) Smart e-health gateway: bringing intelligence to internet-of-things based ubiquitous healthcare systems. In: 2015 12th annual IEEE consumer communications and networking conference (CCNC). IEEE, pp $826-834$

38. Rantz MJ, Skubic M, Koopman RJ, Phillips L, Alexander GL, Miller SJ, Guevara RD (2011) Using sensor networks to detect urinary tract infections in older adults. In: 2011 13th IEEE international conference e-health networking applications and services (Healthcom)

39. Rashidi P, Cook DJ (2009) Keeping the resident in the loop: Adapting the smart home to the user. IEEE Transactions on systems, man, and cybernetics-part A: systems and humans 
40. Rubin SG, Davies GH (1975) Bed blocking by elderly patients in general-hospital wards. Age and Ageing

41. Shnayder V, Chen BR, Lorincz K, Fulford-Jones TR, Welsh M Sensor networks for medical care

42. Suhonen J, Hämäläinen TD, Hännikäinen M (2009) Availability and end-to-end reliability in low duty cycle multihopwireless sensor networks. Sensors 9(3):2088-116

43. Tamura T, Kawarada A, Nambu M, Tsukada A, Sasaki K, Yamakoshi KI (2007) E-healthcare at an experimental welfare techno house in Japan. The Open Medical Informatics Journal

44. Wartena F, Muskens J, Schmitt L, Petkovic M (2010) Continua: The Reference Architecture of a Personal Telehealth Ecosystem. In: proceedings of 12th IEEE international conference on e-health networking applications and services (Healthcom), Lyon, France

45. Yamazaki T (2007) The ubiquitous home. Int J Smart Home 1(1):17-22

46. Zhou Y, Vongsa D, Zhou Y, Cheng Z, Jing L (2015) A Healthcare System for Detection and Analysis of Daily Activity Based on Wearable Sensor and Smartphone. In: 2015 IEEE 12th international conference on ubiquitous intelligence and Computing and 2015 IEEE 12th international conference on autonomic and trusted computing and 2015 IEEE 15th international conference on scalable computing and communications and its associated workshops (UIC-ATC-ScalCom). IEEE, pp 1109-1114

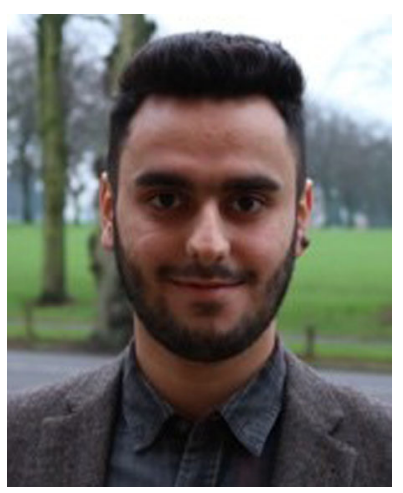

Mohammed Al-khafajiy is pursuing his PhD degree in computer science at Liverpool John Moores University, UK. He received his BSc in Software Engineering from The University of Northampton, and MSc in Software Engineering with distinction from Liverpool John Moores University. His research interests include mHealth, Multimedia tools, Internet of Things, Could Computing and Edge computing.

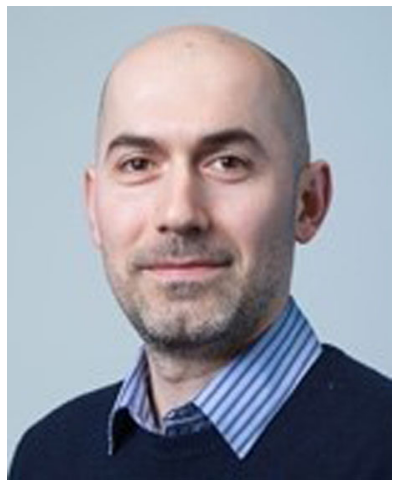

Thar Baker is currently a Senior Lecturer in Software Systems Engineering, and Head of the Applied Computing Research Group, Liverpool John Moores University, U.K. He has published numerous referred research papers in multidisciplinary research areas including: Cloud Computing, algorithm design, SDN, and IoT. He has been actively involved as a member of editorial board and review committees for a number international journals and conferences. 


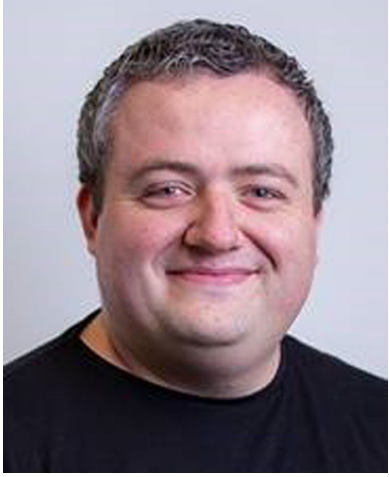

Carl Chalmers is a Senior Lecturer in the Department of Computer Science, Faculty of Engineering and Technology at Liverpool John Moores University. He published many peer reviewed papers in many international conferneces and journals. He has extensive expertise in: Algorithms, Artificial Intelligence and Artificial Neural Network.

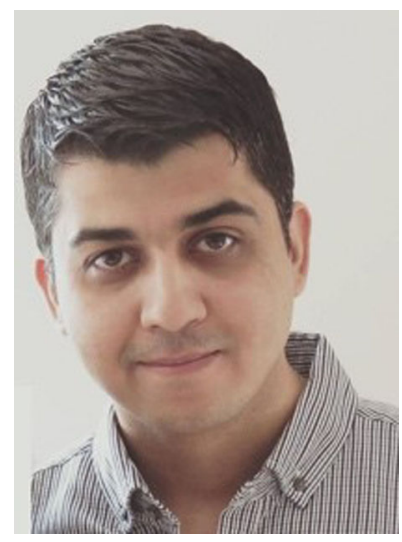

Muhammad Asim is Assistant Professor at the Department of Computer Science, National University of Computer and Emerging Sciences, Pakistan. He obtained his Ph.D from Liverpool John Moores University in the area of wireless sensor networks management. His research interests include Internet of Things, Network Security, and Cloud Computing. Dr Asim has a well-established and promising research track, demonstrated with his wide international publications, conference papers and participation in various conference program committees and journal reviewing. 


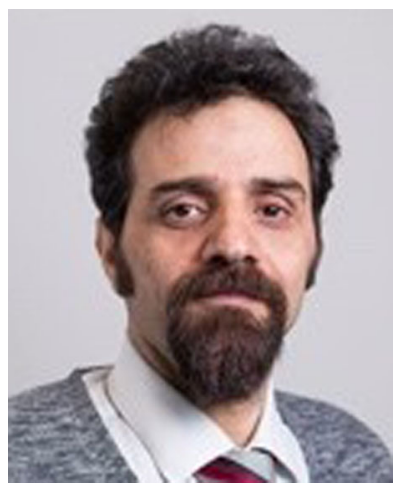

Hoshang Kolivand received his MS degree in applied mathematics and computer from Amirkabir University, Iran, in 1999, and his PhD from Media and Games Innovation Centre of Excellence (MaGIC-X) in Universiti Teknologi Malaysia in 2013. He has completed his Post-Doctoral in Augmented Reality in UTM. Previously he worked as a lecturer in Shahid Beheshti University, Iran and then as a Senior Lecturer in Universiti Teknologi Malaysia. Currently he is a Senior Lecturer in Liverpool John Moores University. He has published numerous articles in international journals, conference proceedings and technical papers, including chapters in books. He is an active reviewer of many conference and international journals. He has also published many books in object-oriented programming and mathematics. His research interests include Computer Graphics, Virtual Reality and Augmented Reality.

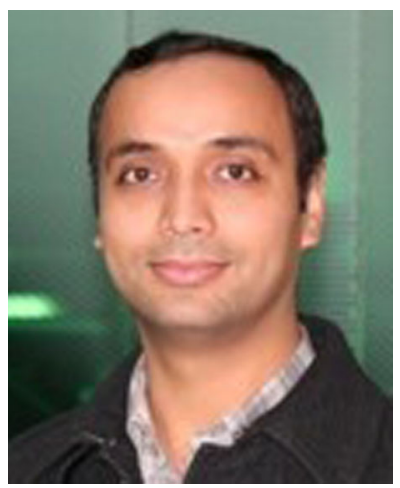

Muhammad Fahim earned his Ph.D. from Department of Computer Engineering, Kyung Hee University, South Korea, in 2014, got Master's degree in Computer Science, specialized in Machine Learning from National University of Computer and Emerging Sciences, Pakistan, in 2009, and obtained Undergraduate degree in Computer Science with distinction "Gold-medalist" from Gomal University, Pakistan, in 2007. He worked as Post-Doctoral research scientist at Ubiquitous Computing Lab, Kyung Hee University, South Korea. He also served as an Assistant Professor in Department of Computer Engineering at Istanbul Sabahattin Zaim University, Istanbul, Turkey and led machine learning research lab for three years. He also worked with Smart Environment Research Group (SERG), University of Ulster, UK and Tecnalia Research and Innovations, Health division, San Sabastian, Spain. He is currently Assistant Professor in Innopolis University, Russia. 


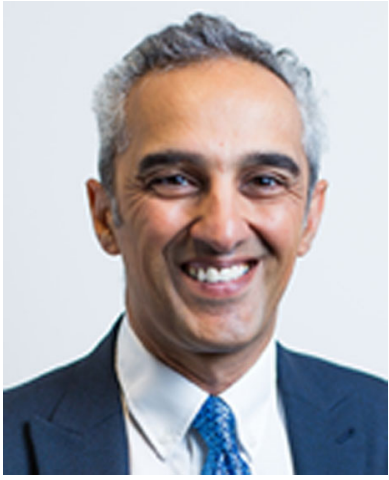

Atif Waraich is the Head of Department of Computer Science at LJMU. He was founder and Director of the Manchester Usability Laboratory from 2006-17 at Manchester Metropolitan University and Enterprise lead for the School of Computing and Mathematics from 2010-17. His research interests cover the use of technology to enhance learning, specifically he is interested in how game like environments can be used to promote learning and to motivate learners to engage in their studies. His other interest relates to human factors and more specifically the use of usability engineering in the development of computer games and immersive technologies.

\section{Affiliations}

\section{Mohammed Al-khafajiy ${ }^{1} \cdot$ Thar Baker $^{1}$ (D) . Carl Chalmers ${ }^{1}$. Muhammad Asim ${ }^{2} \cdot$ Hoshang Kolivand $^{1} \cdot$ Muhammad Fahim $^{3} \cdot$ Atif Waraich $^{1}$}

Thar Baker

T.Baker@ljmu.ac.uk

Carl Chalmers

C.Chalmers@ljmu.ac.uk

Muhammad Asim

muhammad.asim@nu.edu.pk

Hoshang Kolivand

H.Kolivand@1jmu.ac.uk

Muhammad Fahim

m.fahim@innopolis.ru

Atif Waraich

A.I.Waraich@ljmu.ac.uk

1 Faculty of Engineering and Technology - Department of Computer Science, 3 Byrom St, Liverpool L3 3AF, UK

2 National University of Computer and Emerging Sciences, Islamabad, Pakistan

3 Innopolis University, Innopolis, Russia 